\title{
Serpientes u ofidios en el Ecuador
}

\author{
Equipo Editorial[1]
}

1.Práctica Familiar Rural, Ecuador.

Doi: https://doi.org/10.23936/pfr.v5i2.165

PRÁCTICA FAMILIAR RURAL | Vol.5 | No.2 | Julio 2020 | Recibido: 20/07/2020 | Aprobado: 24/07/2020

Cómo citar este artículo

Práctica Familiar Rural. Serpientes u ofidios en el Ecuador. Práctica Familiar Rural. 2020 julio; 5(2).

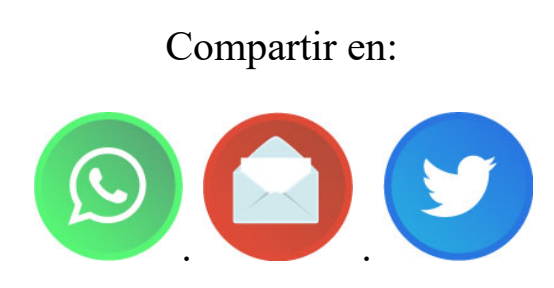

\section{Resumen}

El presente texto ha sido calificado por los editores como un clásico de la medicina, escrito por el Dr. Celín Astudillo, estudioso de las enfemedades tropicales y micosis, que aún en nuestro tiempo, se presenta como un valioso aporte y referente bibliográfico. A continuación, les ofrecemos el capítulo de mordedura de culebra y otros tóxicos, escrito en su libro Aspectos de la Medicina Andina y Tropical del Ecuador publicada en 1977 por la Editorial Universitaria en Quito.

Palabras clave: medicina tropical, serpientes, ofidios, Celín Astudillo.

\section{Snakes and snakes in Ecuador}

\begin{abstract}
The present text has been described by the editors as a classic of medicine, written by Dr. Celín Astudillo, a student of tropical diseases and mycoses, which, even in our time, is presented as a valuable contribution and bibliographic reference. Next, we offer you the chapter on snake bite and other toxins, written in his book Aspects of Andean and Tropical Medicine of Ecuador published in 1977 by the Editorial Universitaria in Quito.
\end{abstract}

Keywords: tropical medicine, snakes, ophidians, Celín Astudillo. 


\section{Nota editorial}

El Dr. Celín Astudillo Espinoza: Nació en la ciudad de Riobamba el 20 de enero de 1914. Estudio en el Instituto Pasteur de París donde hizo una especialidad de enfermedades tropicales y micosis, desde1966 ejerció la cátedra de Parasitología y Enfermedades Tropicales en la U. Central, consiguiendo para la misma que se independice y se le conceda la importancia que le corresponde a esta cátedra, a más de médico, connotado escritor realizo la biografía del padre Juan de Velasco, Dr. Velasco y Dr. Ayora, científico e investigador incansable, de esa generación de médicos como Rodrigo Fierro, Plutarco Naranjo en el ámbito ecuatoriano, inspirados por otras figuras latinoamericanas como Daniel A. Carrión que sabían combinar temas, científicos y humanitarios.

Usando las palabras del Dr. Astudillo, en los años 80, al describir los problemas del estudio de las enfermedades tropicales en la crónica crisis de la investigación científica en el Ecuador se debe al poco interés, el desconocimiento y la ninguna importancia que le dan los gobernantes y las autoridades universitarias a las tareas de investigación, y creen que ser investigador, es una especie de vocación de pocos hombres proclives al sacrificio y enloquecidos por la curiosidad de fenómenos.

Es preciso que la sociedad entera esté informada de los trabajos de investigación, esto no es posible con una furtiva publicación en las cada vez más difíciles editoriales ecuatorianas, publicaciones que han tenido mayor acogida y circulación en el exterior, ya que en el país se ha tratado de minimizar, e ignorar

\section{Serpientes u ofidios en el Ecuador}

Las serpientes son reptiles vermiformes, alargados, cubiertos de escamas, de diferente morfología y cuyos componentes como la cabeza, con sus dientes y huesos característicos, sirven para diferenciarlos y clasificarlos. Ciertas especies de serpientes están provistas de glándulas y dientes especializados para secretar e inocular, una substancia que puede ser inocua o tóxica y venenosa. Presentan generalmente cuatro hileras de dientes superiores, los dos externos son dientes maxilares y los dos internos son dientes palatinos. Los dientes inferiores, se presentan en una sola hilera a cada lado. En las serpientes venenosas, los dientes maxilares son los inoculadores, los cuales están en intercomunicación con los aparatos glandulares. Las glándulas de las serpientes son muy variadas, unas están simétricamente colocadas a cada lado de la cabeza, otras son impares, como sucede con las nasales y lacrimales (serosas) Otras son labiales o linguales que son glándulas mixtas, de éstas, las supralabiales, están ligeramente cubiertas por la piel, siendo de mayor tamaño que las anteriores y su lóbulo medio, constituye la glándula venenosa propiamente dicha. De acuerdo a la morfología y funcionalismo de los dientes y glándulas supe labiales, se clasifican las serpientes en:

1) Typhlopidae, de dientes macizos y glándulas de secreción no tóxica, serosas; a este tipo corresponden las boas.

2) Aglyphas, de las cuales en Sudamérica hay las especies conocidas con los nombres de Boipebas (xenodon), Vacanina (Spilotes), Liophis o Rhadinea, Drimobius, Jararacusu de brejo, etc. Los dientes son también macizos y los posteriores mayores que los anteriores, aptos para deglutir la presa, siendo su secreción altamente tóxica en especial para los pequeños animales. Las cuatro hileras de dientes superiores, causan múltiples heridas, por donde penetra el veneno.

3) Opistoglyphas, siendo su característica que en los dientes posteriores hay un canal que conduce la substancia tóxica de las glándulas supralabiales, las cuales están muy desarrolladas. A estas pertenecen las denominadas culebras verdes y las falsas corales (Philodryas) Oxitropus, Eritrolamprus, la Mussurana; estas serpientes determinan molestias cuando la mordedura lo hacen en ciertos sitios delicados de la piel, por ejemplo, en la zona interdigital, produciendo edema local, eritema, dolor, náuseas, malestar general, de escasa duración.

4) Proteroglyphas, son las serpientes "coral" verdaderas, en las cuales los dientes están atravesados por un profundo canal, que comunica con la voluminosa glándula de veneno, que puede ser en uno o dos pares, 
colocados en la parte anterior de la boca.

5) Solenoglyphas, a esta especie pertenecen las víboras viperinas y crotalinas con un solo par de dientes grandes, aptos para la inoculación y provistos de un gran canal completo, en el cual desembocan las glándulas venenosas, que son muy voluminosas; sus dientes están protegidos por una vaina mucosa y se sitúan a lo largo de la bóveda palatina, los cuales se enderezan y descubren en cuanto la serpiente abre la boca. Estas serpientes son muy peligrosas.

De todas las serpientes sudamericanas, las que determinan el mayor número de accidentes son las Crotalinaes, del grupo de las La Mussurana (Oxiropus cloelia), mide hasta dos metros de largo, siendo su color negro azulado y se alimenta de otras culebras viperinas. Las Boidae son muy desarrolladas y tienen una variada sino nimia como: boa, giboiayagua, cabeza de perro, etc. Las boas tienen una cabeza triangular cubierta de escamas, lo mismo que el cuerpo y la cola. Los dientes son muy agudos y desarrollados, determinando mordeduras dolorosas, aunque no mortales. Las Eunceles murinas de la región amazónica, miden hasta 12 metros y trituran para tragar sus presas, de mediano tamaño. Las Colubriadae comprenden todas las variedades comúnmente denominadas culebras, de las cuales hay únicamente dos especies venenosas, que son las Hidropinas y las Micririnaes o Elapinae (Coral). Por su dentición las culebras se dividen en tres grupos: Aglyphas, Opistogliphas y Proterogliphas. Las primeras se las encuentran en el campo, otras en los árboles de las regiones tropicales y subtropicales, frecuentemente se las confunde con la yararacussi venenosa, son de color marrón amarillento, con manchas laterales obscuras (Drymobius bifo, satus).

Las denominadas Xenodón, son parecidas a las Bothrops y a las Yararas, de color amarillento, con dibujos triangulares; cuando se encolerizan se achatan y dilatan el cuello, sus colmillos no son acanalados. Las culebras Opistoglifas de la variedad Philodrias, son arborícolas, su cuerpo es delgado y la cola larga, de color verde, se les llama por esto, culebras verdes, culebras de bejuco, son agresivas y producen en el hombre accidentes locales, en el sitio de la mordedura, siendo sus dientes posteriores acanalados. La Eritrolamprus aesculapi, presentan un fondo rojo con anillos negros, los que varían de 10 a 40; su mordedura produce edema local, con dolor y temperatura elevada.

Las Proteroglifas, son serpientes venenosas y en Sudamérica hay dos variedades que son: la Micrurinae, de cuerpo cilíndrico y la especie marina Hidropinae, de cuerpo achatado lateralmente, son aptas para la natación. Las culebras Micurinae, son las víboras de coral, cuyo color es rojo, blanco y negro, distribuidos en anillos alternos y comprenden unas 30 especies, habiéndose conformado un grupo especial, con las especies de corales andinas las Micruroides, que presentan como característica, el color rojo triángulos negros laterales como la Micruroides narducii, siendo su veneno semejante al de la culebra Micrurus. La serpiente marina Hidrus platrus que se encuentran en las costas del Pacífico desde Panamá hasta el Perú, se las halla en las rocas cubiertas de algas o en alta mar. Las Solenoglyphas, tienen en América una variedad de serpientes sumamente venenosas, como es la Crotalinae, conocida también con el nombre de Víbora de las cuatro narinas y comprende cinco géneros, caracterizados por que en la extremidad de su cola, poseen algunas de ellas, el cascabel, que es un aparato formado por unos anillos córneos y al cual deben su nombre de "víbora de cascabel", que además presentan en su cabeza, unas escamas, con una disposición especial, o también unas placas grandes.

Las serpientes Crotalinae son nocturnas, ovovivíperas, con excepción de las del género Lachesis muta que pone huevos del tamaño de los de la gallina y de color rosado. Estas serpientes, tienen una apariencia distinta, en cuanto al dibujo, color y disposición de las manchas del dorso, en las distintas regiones sudamericanas y su veneno es de características neurotóxicas; algunas de ellas viven en los campos secos, otras en los bosques; son de cuerpo grueso y pesado, salen por la noche a cazar pequeños roedores, son muy irritables, se despiertan al menor ruido y hacen sonar su cascabel, mordiendo a todo organismo, que se encuentra cerca.

Género Lachesis. Tiene diversos nombres populares como el: Surucucú o Verrucos (como se llama en el Ecuador) y llega a medir hasta 2,50m., es de color claro, con manchas triangulares o romboidales, negras, de 
escamas gruesas y salientes. Se esconden debajo de las hojas secas. Es grande la cantidad de veneno de que está provista, hasta un gramo de veneno seco y sumamente activo.

Género Botrops. Tiene una gran cantidad de especies, siendo las principales las del grupo: Botrops atrox, de gran incidencia en América y que en el Ecuador y países vecinos lleva el nombre de Equis(X) o de Rabihueso, Rabo amarillo, Terciopelo, etc. y mide hasta 1,80m., es de color gris con manchas triangulares grises o negras y a los lados de la boca hay una faja longitudinal negra y otra amarilla, vive en las selvas, cerca del agua y es muy agresiva. Su veneno es fuertemente neurotóxico y necrosante. La Botrops jararacussú, mide más o menos dos metros de largo, su color es amarillo, con grandes manchas triangulares negras, es muy agresiva y toda su variada sinonimia se hace a base de su color amarillo característico. En el Ecuador, según el estudio del Dr. Sotomayor Navas, se han estudiado unas 36 especies del género Botrops y son las más abundantes entre las serpientes venenosas de nuestros campos y también reciben los nombres de Equis jerga, Equis pachona, y en general el nombre de Equis, es el más común por esas sus franjas que en forma de una X, cruzan sobre el lomo. Las denominadas culebras "Tigre", o "Sobrecamas", son de gran tamaño, pertenecen a la familia "Boas", género Phyton y sin embargo de que el pueblo le da el nombre de "Mata caballo", no son venenosas. Las glándulas de veneno de las Botrops, que han sido estudiadas en el Ecuador, son muy grandes, del tamaño de un frejol y su conducto excretor, desemboca en la base del diente inoculante y se encuentra subcutáneamente, entre el ojo y el ángulo posterior de la cabeza y en la porción posterior de la glándula, hay un músculo que le comprime, en el momento de la expulsión del veneno, el cual es de color amarillo limón o anaranjado.

Las verdaderas culebras Coral, del género Microrurus (Microrurus fuivis, Microrurus corallinus), no abundan en los campos del Ecuador, pero en las que hay, se ha observado que su veneno es muy activo y tienen acción neurotóxica. (El veneno de una serpiente de Malasia, la Agkistrodon rhodoston, puede combatir la trombosis humana, por su efecto anticoagulante; en un Laboratorio farmacéutico italiano, se ha logrado separar el veneno propiamente dicho, de la substancia anticoagulante, de alto poder curativo. Actúa sobre el fibrinógeno, del que se deriva la fibrina, que es el elemento estructural del trombo. Se está utilizando como remplazo de la heparina).

\section{Sintomatología producida por la mordedura de las serpientes.}

Hay tres clases de accidentes en tres períodos principales:

1) Primario, con repentino shock inicial;

2) Período agudo o de estado, con alteraciones sanguíneas, hemorragias, accidentes nerviosos generales y locales, shock secundario y

3) Período tardío, con fenómenos tóxicos y lesiones locales. La muerte se puede observar, en cualquiera de los tres períodos, según la cantidad de veneno inyectado y la resistencia del individuo.

El shock del período primario depende en gran parte de la vía de penetración del veneno, si es en una región muy vascularizada, determina una absorción rápida del veneno y una respuesta rápida, el shock se acompaña de caída de la presión arterial, con alteraciones sanguíneas y visuales, fibrilación de los músculos de la cara, nistagmus, convulsiones epileptiformes, etc. Más o menos después de una hora de la mordedura de la culebra, se instala el cuadro clínico completo, con algunas lesiones sanguíneas como la incoagulabilidad de la sangre, la leucopenia y eritropenia Inicial, que rápidamente da lugar a la producción de nuevas formas globulares con predominio de neutrófilos, mielocitos, formas juveniles. Se presentan hemorragias múltiples, especialmente por la boca, la nariz, intestinos, riñones, etc.

Las manifestaciones nerviosas están especialmente relacionadas con la visión. Hay parálisis musculares de los miembros, tanto inferiores como superiores, los de la nuca. Son frecuentes las perturbaciones de la deglución y de la fonación y casi todos los órganos se encuentran trastornados. Generalmente se produce una hipotermia marcada.

Alrededor de los puntos de mordedura se presenta una intensa congestión, luego edema hemorrágico que se extiende a todo el miembro afectado, al tronco y a todos los músculos cercanos, estos edemas por 
compresión de los órganos vecinos se presentan sumamente dolorosos. En el período final se agravan los síntomas respiratorios, los urinarios con fuerte albuminuria, cilindruria, oliguria y hematuria. Hay trastornos hepáticos, del bazo, de los órganos hematopoyéticos, están fuertemente alterados los reflejos, la sensibilidad y la temperatura. Tratamiento.

La sueroterapia antiofídica es el tratamiento de elección, sin embargo, en ciertas circunstancias se continúan utilizando algunos tratamientos tradicionales encaminados a evitar la difusión del veneno, para destruirlo o neutralizarlo en el sitio de la mordedura; también para el tratamiento de las complicaciones. Se ha calculado que hasta un $30 \%$ de las mordeduras de serpientes curan espontáneamente o sin tratamiento seroterápico.

Para evitar la difusión del veneno o para destruirlo, desde tiempos inmemoriales se han utilizado los procedimientos de la ligadura, la succión, la cauterización y amputación del miembro afectado. El tratamiento local, con substancias quimioterápicas no dan resultados prácticos.

Seroterapia. El tratamiento seroterápico se inició con los sueros preparados por Calmette en 1894 contra el veneno de la Cobra naja tripudians. Luego se prepararon los sueros antiofídicos polivalentes, algunos de ellos de eficacia especialmente regional (Regiones africanas, regiones asiáticas y americanas). Los sueros preparados por Calmette eran a base de inyecciones de venenos atenuados por cloruros o hipocloritos y luego continuando con inyecciones de venenos puros. Los sueros utilizados actualmente son preparados en caballos y luego purificados o concentrados.

Un suero se titula según su poder neutralizante o curativo, para lo que hay diferentes métodos utilizados en los diferentes Insti tutos. Algunos sueros conservan su actividad durante muchos años, según la inmunización de los animales de donde provienen, dándose casos de conservación hasta por 10 y 15 años; pero generalmente el plazo de actividad es de 2 a 4 años.

Respecto a las mordeduras de serpientes en la zona tropical del país, el Dr. Teodoro Maldonado Carbo, de Guayaquil ha realizado observaciones en 4.803 casos, en el Hospital "Luis Vernaza", en el período de tiempo comprendido entre el año 1900, hasta el 30 de noviembre de 1957, cifra que nos da la idea de la gravedad del problema del ofidismo en nuestro país; pero es necesario indicar que esta estadística es únicamente de enfermos atendidos en el Hospital Luis Vernaza de Guayaquil y hay un número igual o mayor que se someten a tratamientos en instituciones particulares o en sus mismos recintos, como también hay un sinnúmero de siniestrados o mordidos de serpientes en las demás provincias del Litoral Ecuatoriano, en los valles interandinos y en la Región oriental. Se anota en la estadística del Dr. Maldonado que a partir del año 1950 se incrementa el número de mordeduras de serpiente, lo que se explica por el intenso desarrollo que tuvo desde ese año el cultivo del banano y que en el interrogatorio de los pacientes, siempre responden que "fueron mordidos por las víboras en los platanales".

De la cifra de 4.803 mordidos por las serpientes se han producido 189 defunciones, lo que nos da un porcentaje de 3.95\% En el Ecuador se hace obligatorio el uso del suero antiofidico para combatir los accidentes del ofidismo desde el 19 de noviembre de 1935, según Decreto Supremo, que fue modificado el 9 de diciembre de 1937 por otro Decreto Supremo del General Alberto Enriquez Es necesario insistir en la obligatoriedad de arbitrar los me dios de profilaxis contra el ofidismo, para lo que es necesario recomendar el uso de vestidos especiales sobre todo evitando la desnudez de pies y piernas que son los sitios de predilección y mayor porcentaje de las mordeduras.

Hay que educar al campesino a que evite la metedura de sus manos a los matorrales, en los depósitos de hojarasca y demás sitios en donde comúnmente anidan las serpientes, utilizando los artefactos que se han inventado para el caso, llamados ganchos o garabatos. servicio médico antiofídico del Hospital Luis Vernaza se han empleado como terapéutica rutinaria la inyección de $20 \mathrm{ce}$, de suero antiofídico a todo enfermo que llega, "haya sido o no inyectado en el lugar del accidente". Después con el estudio del caso clínico se repite la dosis o se le aumenta a 40 o $60 \mathrm{cc}$, de suero.

Los sueros deben conservarse al abrigo de la luz y del calor, para conservar mejor su actividad. El tratamiento con el suero debe efectuarse lo más rápidamente posible, si se lo hace dentro de tres horas el 
resultado generalmente es favorable, aun es factible hasta a las seis horas, pero más tardíamente se corre el riesgo del fracaso. La dosis debe ser elevada hasta de 30 centímetros cúbicos y si los síntomas no disminuyen en tres horas, se debe repetir la dosis. En caso de recaída, también es indicado repetir la dosis de suero.

Generalmente se utiliza la vía hipodérmica (inter escapular) pero en casos graves o tardíos se puede utilizar la vía intravenosa, al es posible diluyéndolo en uno o dos volúmenes de suero físiológico o en solución glucosada hipertónica. Para evitar los accidentes séricos, es aconsejado mezclar el suero en la misma jeringa, con $0,5 \mathrm{~cm}$. de adrenalina al milésimo. La acción del suero se hace presente, desde las tres horas más o menos después de su Inyección, se completa el tratamiento con la aplicación de medicamentos sintomáticos como por ejemplo in aplicación de analépticos circula torios o respiratorios. 